\title{
Large scale field tests of high-tensile steel wire mesh in combination with dynamic rockbolts subjected to rockburst loading
}

\author{
R. Bucher Geobrugg Australia Pty Ltd, Australia \\ M. Cala AGH University of Science and Technology, Poland
}

A. Zimmermann Geobrugg AG, Switzerland

C. Balg Geobrugg AG, Switzerland

A. Roth Geobrugg AG, Switzerland

\begin{abstract}
More and more underground mines are experiencing rockburst, which is a stress-induced violent ejection of rock that is normally encountered in deep mines. There are different approaches to determine the energy from rockburst, which is caused by excess stress concentrations in the rock mass. A major engineering challenge for mines experiencing this significant seismicity is to design the dynamic performance of their support systems. Therefore, a retaining system using dynamic rockbolts in combination with high-tensile steel wire mesh was tested to ascertain the behaviour and quantify the suitability of the system. A special large scale test facility was constructed for the purpose, which allowed verification of the percentage of energy transmitted by the rockbolts and wire mesh, based on the input energy. Numerous tests were carried out providing first findings on the load distribution within the support system.
\end{abstract}

\section{Introduction}

As more and more near surface deposits are mined out, deeper mines are required in order to continue the exploitation of resources. This inevitably leads to the occurrence of more rockbursting, deformation problems and safety issues. Rockburst means stress induced loosening of parts of the rock mass under enormous energy release. It seems to be similar to damage from natural seismic phenomena. Rockburst events occur mostly in deep tunnels and mines but also in regions with high horizontal stresses.

There are different methods to mitigate rockburst risks thus reducing exposure of personnel. Changes include mine design, layout, and extraction sequence (Potvin, 2012). In addition the ground support needs to be chosen and designed in such a way that it can cope with the conditions. In deep mines the ground support is complex and cost is high. Static ground support is not satisfactory for such a demanding environment. The ground support consists of the rock reinforcement, e.g. bolt, a surface support, e.g. mesh, and the connection between the two, e.g. plate. For dynamic loads it is essential that these components fit and work together as a system. The observations of Heal (2007) show that most of the damage done from rockbursting led to the failure of the surface support or the rupture of the reinforcement. In order to investigate the dynamic behaviour of reinforcement elements and surface support extensive testing was carried out at various test sites. Some tests were done in South Africa, Canada and Australia. Hadjigeorgiou and Potvin (2011) put the results of the various tests together and tried to make them comparable. However, the boundary conditions of the test sites were different making comparison difficult. There are two active test sites at the moment; one is in Canada at CANMET and the other in Australia at WASM (Player et al., 2004). The WASM test site is the best instrumented facility and is based on the momentum transfer concept. It can test reinforcement, surface support and to a certain extent combinations of the two. 


\section{Motivation}

Geotechnical engineers design support systems for their operations, based on the work done in Canada which resulted in the Canadian Rockburst Manual (Kaiser et al., 1995) and the above mentioned testing of reinforcement and surface support. It is widely known that all elements of the support scheme should work together, but there is little research data available on the dynamic interaction of the main components. In many cases the energy absorption capacities are added together in a superposition for obtaining the capacity of the system as a whole.

Both in static and dynamic loading it is important that the surface support can bear the load of the rock mass and transfer it to the reinforcement. Furthermore, in dynamic loading the reinforcement should start yielding in order to absorb energy without the surface support failing beforehand. Heal (2007) comes to the conclusion that using the dynamic capacity of the reinforcement alone for the design would likely overestimate the capacity of the system. Potvin (2012) therefore proposes to look at the weakest link of the support system and since this is in most cases the surface support, makes the assumption to use the capacity of the surface support as the capacity of the system.

In this study it is believed that the above mentioned approach is too conservative and that more data on the interaction between the reinforcement, the surface support and the connection elements is required. This is one reason for using the test site in Walenstadt, Switzerland, where a $3.6 \times 3.6 \mathrm{~m}$ surface support can be dynamically tested with four dynamic rockbolts in a regular bolt pattern. The other motivation comes from the need to translate the results of the individual component tests at test sites like the WASM site (Player et al., 2004) into full-scale tests for proving the suitability and fitness for use of the overall system. Another option is to use blast tests for this (Heal et al., 2004), but there the loading is questionable and the testing is hardly repeatable.

The hypothesis of this work is based on the assumption that during dynamic loading of a surface support scheme, the surface support is loaded by a part of the fractured mass. The resulting force in the surface support will then be transferred to the connection element, e.g. plate, and then on to the reinforcement, with the other part of the load going directly to the reinforcement. The rock mass around the bolt is bonded to the bolt and held by the plate. There will be an arching effect in the rock mass in between the bolts, depending on the fracturing. Consequently part of the loading will go directly on the bolts and not on the mesh. To verify the difference in loading, load cells were used in the test set-up at both ends of the bolts. It is the aim of the test setup to measure the loads in the different elements and then back calculate what load was borne by the surface support and how much was carried by the dynamic reinforcement directly. This is of course dependent on the type of rock mass and cannot be seen as a general ratio.

For these large scale field tests and their analysis, the calculation of the energy output during a rockburst event is the main topic. Additionally important is the development of adequate retaining elements, such as reinforcement and surface support. Their behaviour during rockburst has to be proven and their interaction has to be observed and described as well. The test construction should be able to test this so called interaction and show how much energy will be absorbed by reinforcement, surface support and the test set-up itself. This may be compared to the breakage of the rock in a real rockburst event which also absorbs energy.

\section{$3 \quad$ Large scale field tests}

Many tests regarding the behaviour of dynamic reinforcement and surface support alone have been carried out already. Thus the behaviour of the individual retaining elements is well known. But for safety in tunnelling and mining projects it is of great interest to have a complete support scheme, which protects the underground workers from risks, such as rockburst. This large scale field tests are supposed to show the behaviour of dynamic high-tensile mesh and dynamic rockbolts installed together. The tests should indicate the load and energy distribution between the components. 
The main goal of these trials is to evaluate a dimensioning concept, where the retaining elements can be designed for dynamic conditions. These calculations are based on the energy distribution between the reinforcement and the surface support. In this project, research for the energy release during rockburst phenomena is not considered. The input energy for the tests has been assessed and classified based on the dynamic tests conducted at WASM (Thompson et al., 2012).

\subsection{Test set-up}

The main frame of the test set-up is made of steel, with a high tensile chain link mesh horizontally connected to the frame by lacing wire rope through mesh and frame (Figure 1). The mesh is hold back by four dynamic rockbolts in a typical pattern.
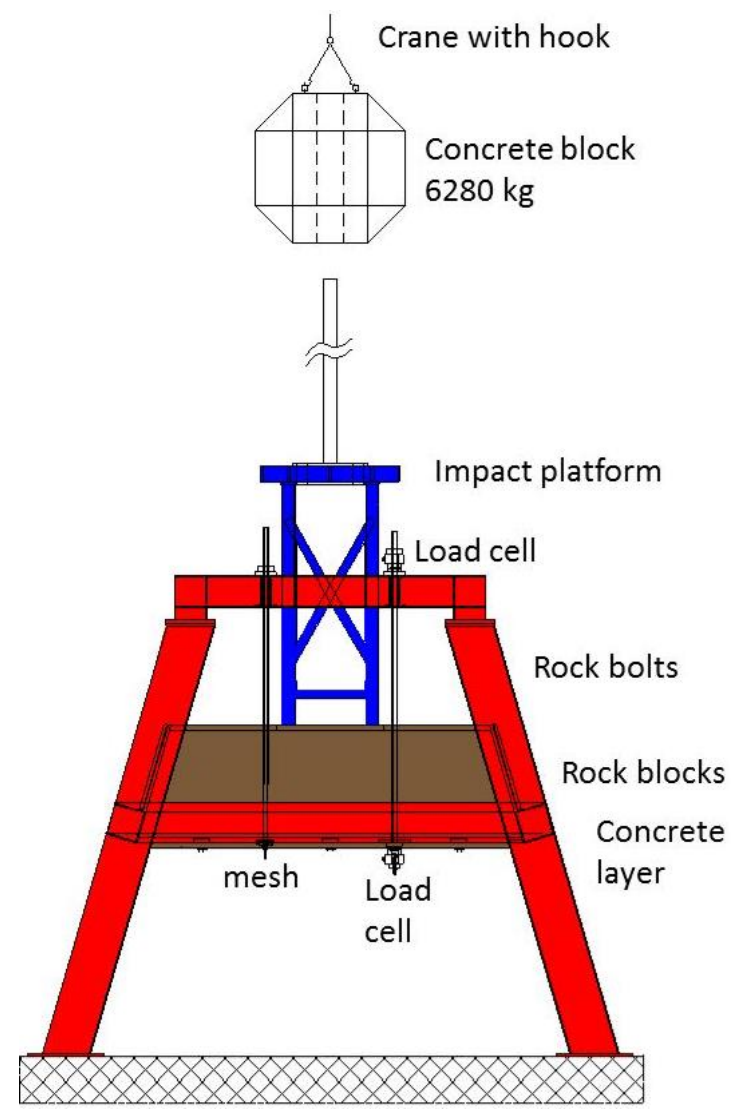

\section{Figure 1 Test set-up}

Formwork was installed underneath the wire mesh. A plastic foil was placed on top of the wire mesh, before concrete was poured onto the formwork. The plastic foil was used to keep the mesh separated from the concrete slab. Before the tests were conducted, the plastic foil was removed. Natural rock boulders and an equalizing layer of gravel were then placed on the top of the thin concrete slab. This layered build-up of concrete, rocks and gravel is intended to simulate the pre-fractured rock mass prior to the rockburst, with the concrete plate as a base to prevent mesh and bolts from preloading. The concrete slab is designed to crack under load as there is no reinforcement in the concrete. On top of the gravel layer, a load distribution plate is installed to initiate the energy impact caused by the test block's impact. The test block is lifted by a crane. To start the test, the crane will disengage the test block which then falls onto the impact platform on the top of the construction. The impact platform on the steel stamp is connected with the load distribution grid lying on the equalising gravel layer.

While following physical laws, the energy impact on the whole construction is the difference of the potential energy of the test block before and after the impact. The maximum height to lift the test block is limited by the length of the guiding steel column to approximately $3.25 \mathrm{~m}$ (Figure 2). The mass of the test block used is $6,280 \mathrm{~kg}$. 


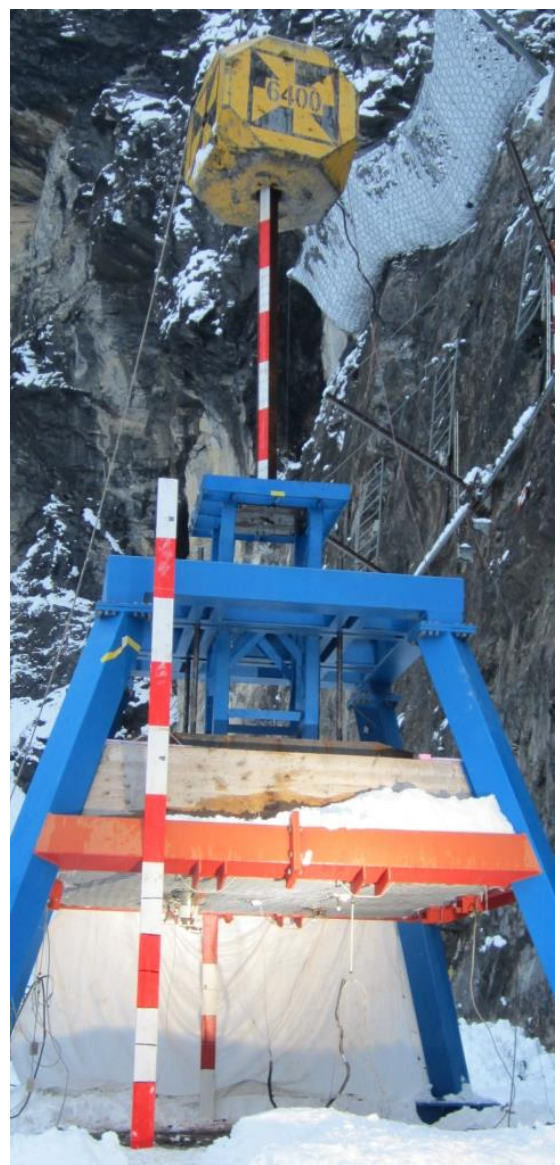

\section{Figure 2 Set-up ready for testing}

For the rockburst trials, a high tensile steel wire mesh of $3.6 \times 3.6 \mathrm{~m}$ (mesh type TECCO G80/4, aperture $80 \mathrm{~mm}$, wire diameter $4 \mathrm{~mm}$, wire strength 1,770 MPa) and four dynamic rockbolts of $3 \mathrm{~m}$ length (bolt type D-Bolt, diameter of $20 \mathrm{~mm}$ ) were used. The dynamic bolts have a deformable length of $1.5 \mathrm{~m}$ and according to the manufactures' specification would elongate by about $15 \%$, meaning an elongation of about $225 \mathrm{~mm}$ is possible. The bolts were grouted into steel tubes (wall thickness $8 \mathrm{~mm}$ ) which are separated into two parts. This gives a defined position for the elongation of the bolt (simulating the detachment point of the fractured rock mass). For the load transmission from the mesh to the bolts, special spike plates are used whose geometry fit exactly for the diamond shaped TECCO mesh. The spike plates ensure that six wires are always grasped by each plate.

\subsection{Instrumentation}

In order to determine factors affecting the system, devices including load cells, high speed cameras and accelerometers were installed to evaluate the distribution of the forces on the mesh, bolt and system as a whole.

The forces and the elongation of the bolts as well as the deflection of the mesh are the primary factors used to calculate the energy absorption capacity of the mesh and the bolts. Based on the results from the test, the energy capacity of the elements can be calculated by the area under the load-displacement curve. By knowing the input energy calculated by the potential energy difference of the test block before and after the impact, the energy absorbed by the test set-up can be back calculated. The procedure is explained in Section 3.5.

The high speed cameras and accelerometers generate measurements of displacement, velocity and acceleration. Therefore, the measurements are redundant and can be cross checked. 


\subsubsection{Load cells}

Two load cells were installed on one of the four dynamic bolts, one cell at the collar of the bolt, the other one at its end (Figure 3). The upper cell measures the total force on anchor and mesh together while the lower cell only measures the vertical load taken up by the mesh.
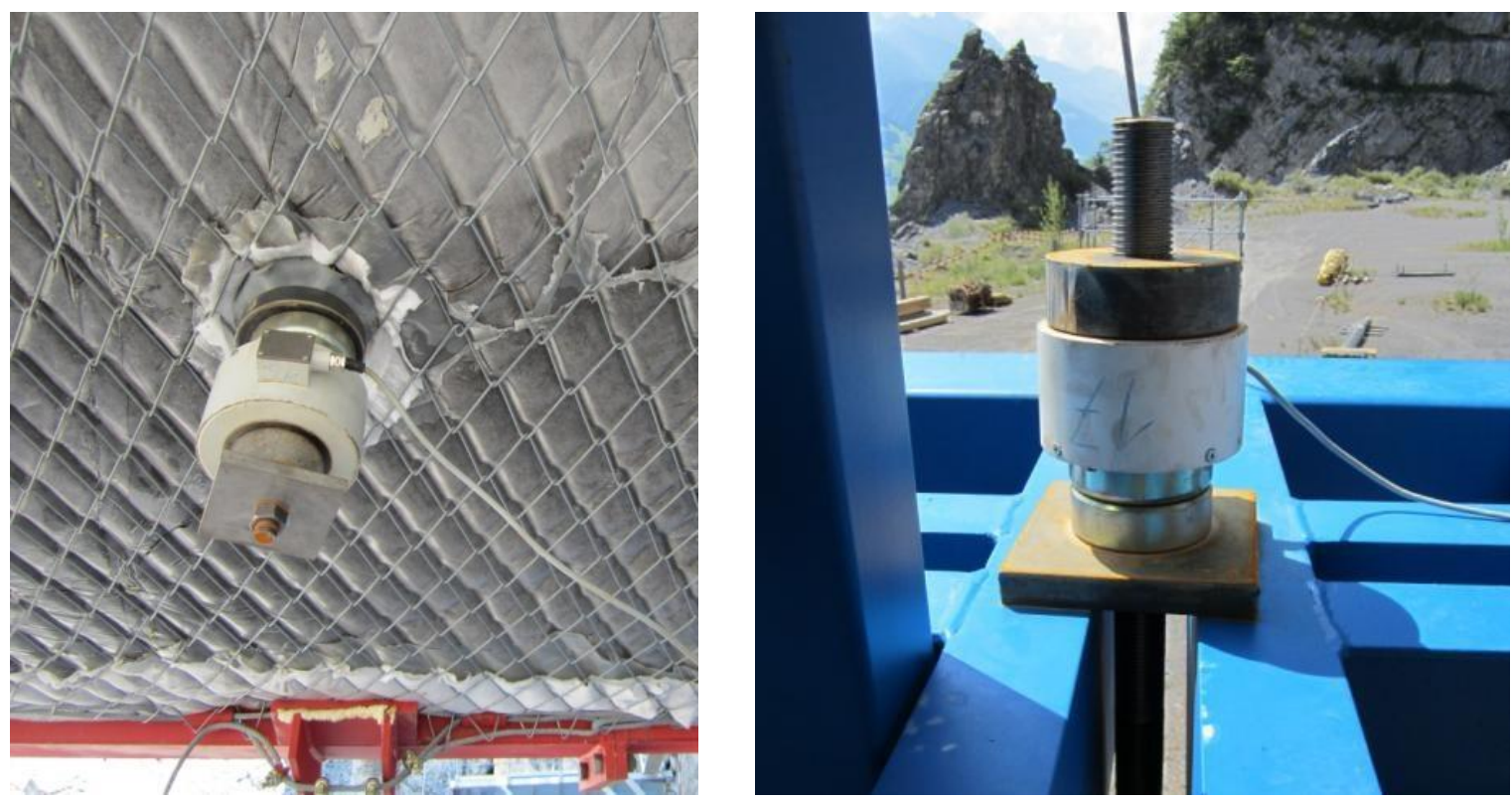

\section{Figure 3 Lower (left) and upper (right) load cell}

\subsubsection{High speed cameras}

Two high speed cameras were installed in front of the steel frame, from where several measuring points on the bolts, the mesh and the test block were filmed. With a computer tracking program the displacement of mesh and bolts can be evaluated out of the grouped pictures per time by pursuit of a measure point on the pictures. The velocity can be calculated by the first differentiation of the displacement and the acceleration by the second differentiation of the displacement. An upper camera films the movement of the test block and the impact whilst a lower camera films the deformation of the mesh and the bolts.

\subsubsection{Accelerometers}

The accelerometers measure the acceleration in time. By a double integration, the deformation can be calculated. This is used to cross check the data output from the high speed camera. Sensors are fixed to the mesh, bolts, the test block and the impact platform. The mesh sensor is able to measure a three dimensional movement of the mesh (Figure 4). 


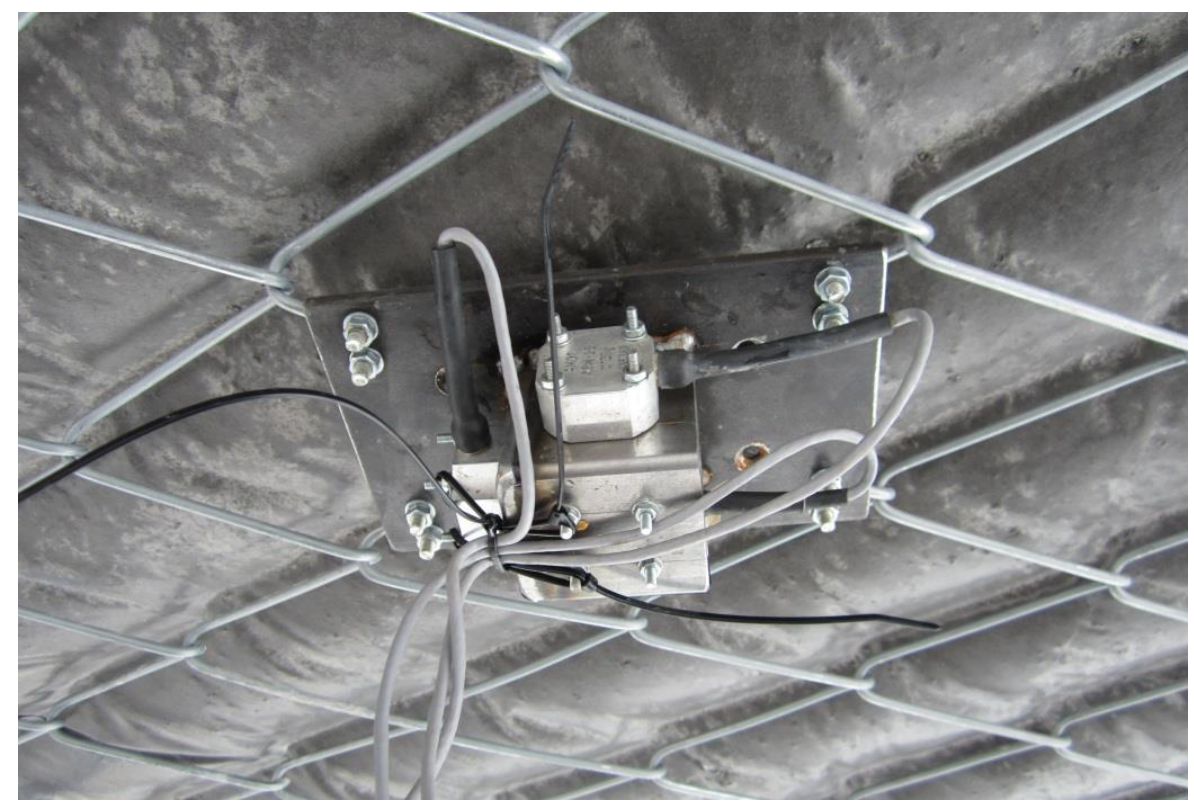

\section{Figure 4 Accelerometer fixed in the middle of the mesh}

\subsection{Test execution}

As already mentioned in Section 3.1, the rockburst is simulated by releasing a heavy concrete test block in free fall on the test set-up. Due to the potential energy difference of the test block before and after the impact, a certain amount of energy is put into the system. This input energy is absorbed by the bolts, the mesh and the test set up itself, including breakage of the concrete slab (Figure 5).

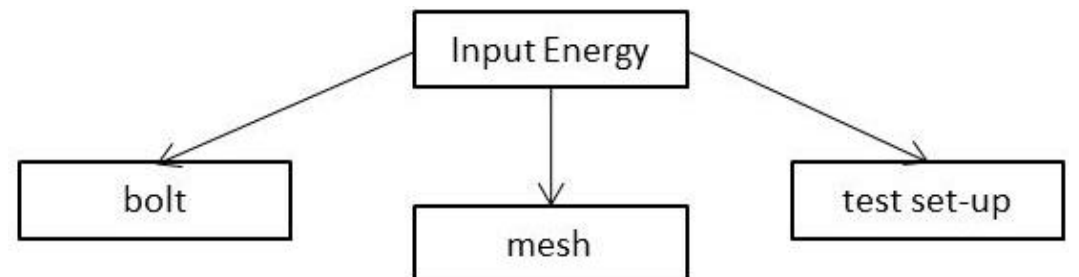

\section{Figure 5 Division of the input energy}

After the dynamic movement of the rock mass, the effect of plastic elongation and deformation of bolts and mesh is easy to see. All the extra elastic deformation can be discovered by analysing the data output from high speed cameras and accelerometers. This will show the maximum deformation of the support elements during the dynamic impact.

Different tests were carried out with various stiffness of the assembly, mainly by varying the thickness of the concrete slab and the assembly of the natural stones which are build-in more or less interlocked. Those adjustments resulted in different loading conditions of the anchors and the mesh which directly refers to variable behaviour of the rock mass in reality.

The following pictures show the plastic deformation of the mesh and the bolts - firstly for a stiff assembly, secondly for a softer one.

For the stiff set-up, the elongation of the bolts is very well seen (Figure 6). The elongation was $150 \mathrm{~mm}$, or $10 \%$ of the dynamic length of the bolt. As noted above, the bolts used in this test are able to elongate about $15 \%$ of their elongation length. 


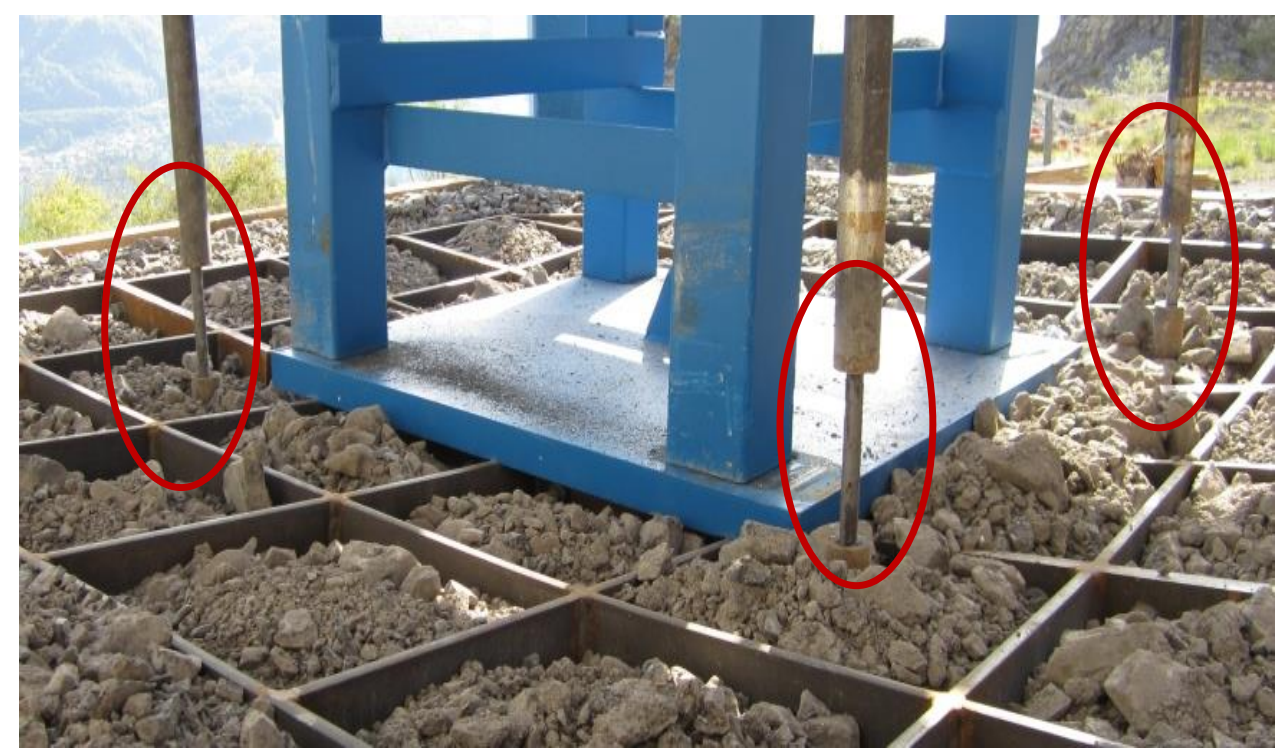

Figure 6 Elongation of anchors for a stiff assembly

For the stiff assembly, the deflection of the mesh was about $140 \mathrm{~mm}$ which is quite moderate (Figure 7). Due to the thickness and therefore stiffness of the concrete slab, the slab only broke up into a few large pieces. Therefore, the loading on the mesh was not in a parabolic shape as expected with more fragmented rock. The shape had a more triangle character due to the big concrete pieces pressed into the mesh. This loading results in higher forces on the bolts compare to the forces on the mesh and therefore, in combination with the major deformation of the mesh, to more energy absorption by the bolts than by the mesh.

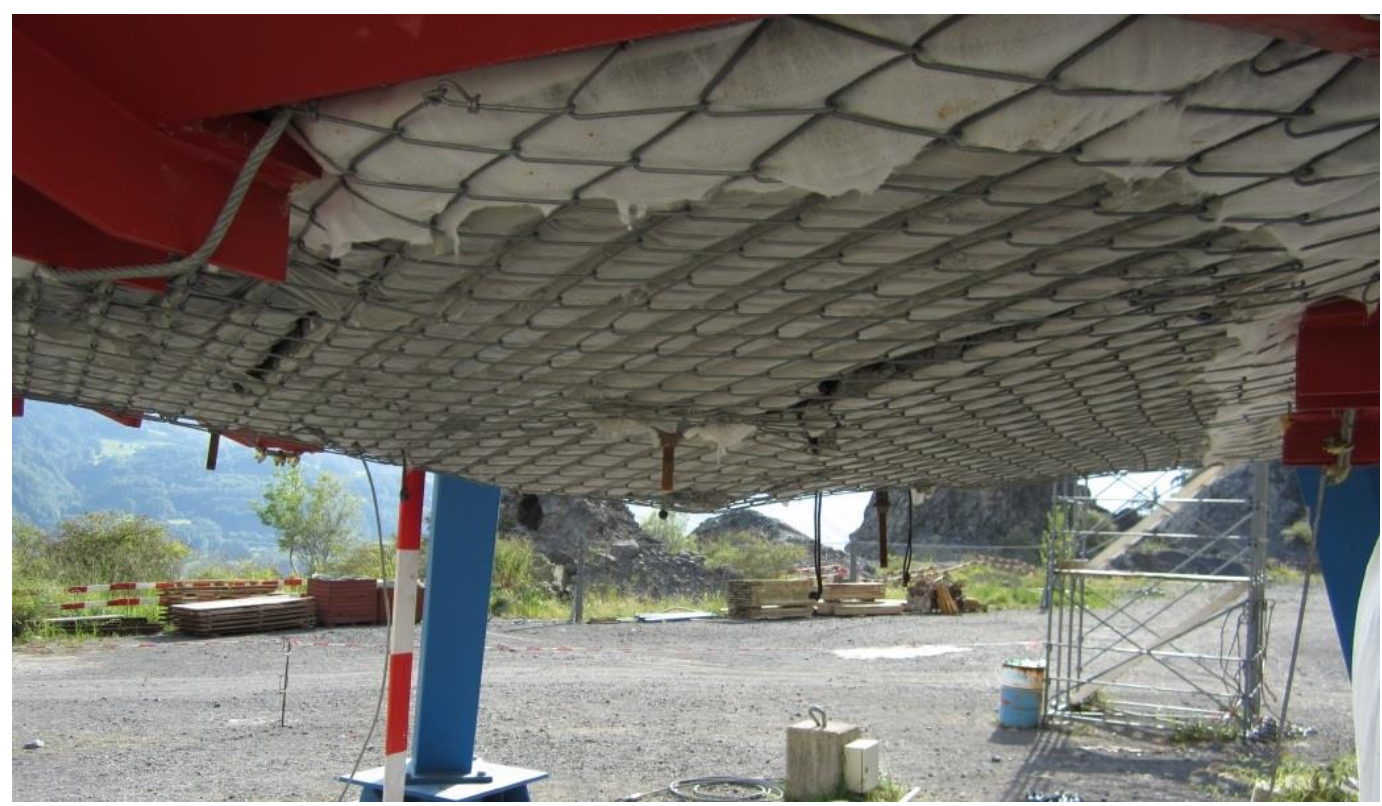

\section{Figure 7 Pressing of the concrete plate into the mesh for a stiff assembly}

In comparison, when the test was set-up with a thinner and less stiff concrete slab, the loading on the mesh resulted in a more parabolic shape, due to the higher fracturing rate in the concrete. As a consequence, the plate and bolt only see the loading from the material directly above the bolt and plate, having a cone shape. In this test set-up the mesh absorbs more energy than the bolts. Figure 8 shows the movement of the bolts of about $25 \mathrm{~mm}$ for the softer set-up. 


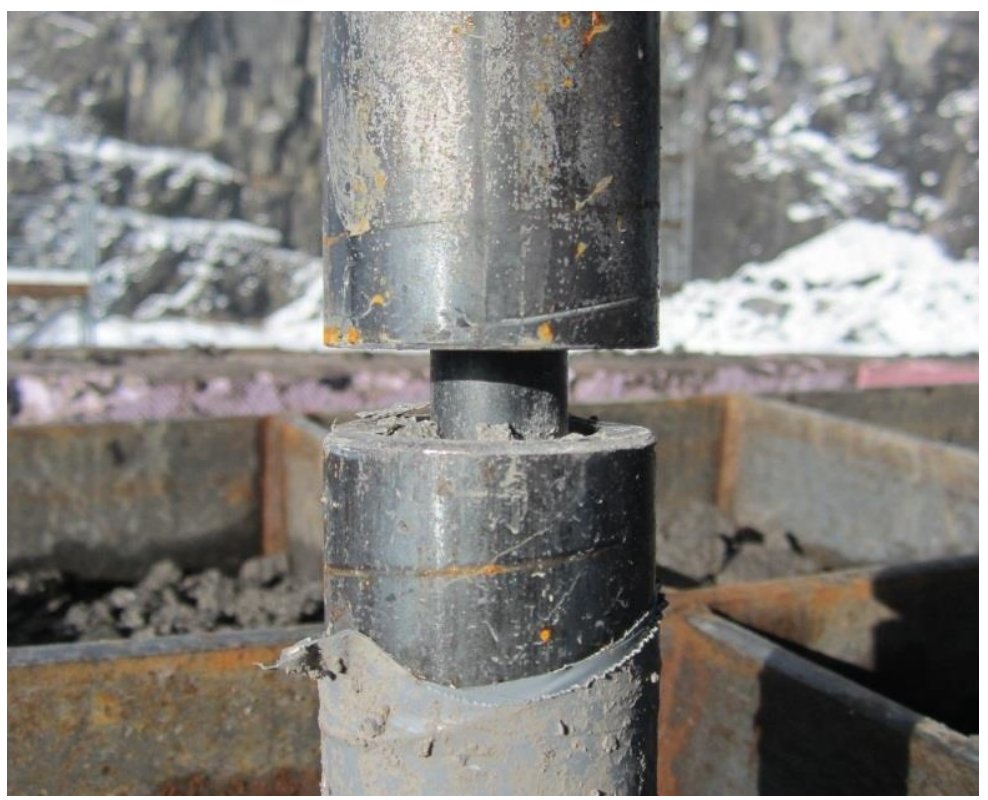

\section{Figure 8 Elongation of anchors for a softer assembly}

The nearly parabolic shape can be noticed, when looking at the deflection of the mesh of the softer set-up (Figure 9 and 10). At its highest deformation, the deflection of the mesh is around $300 \mathrm{~mm}$.
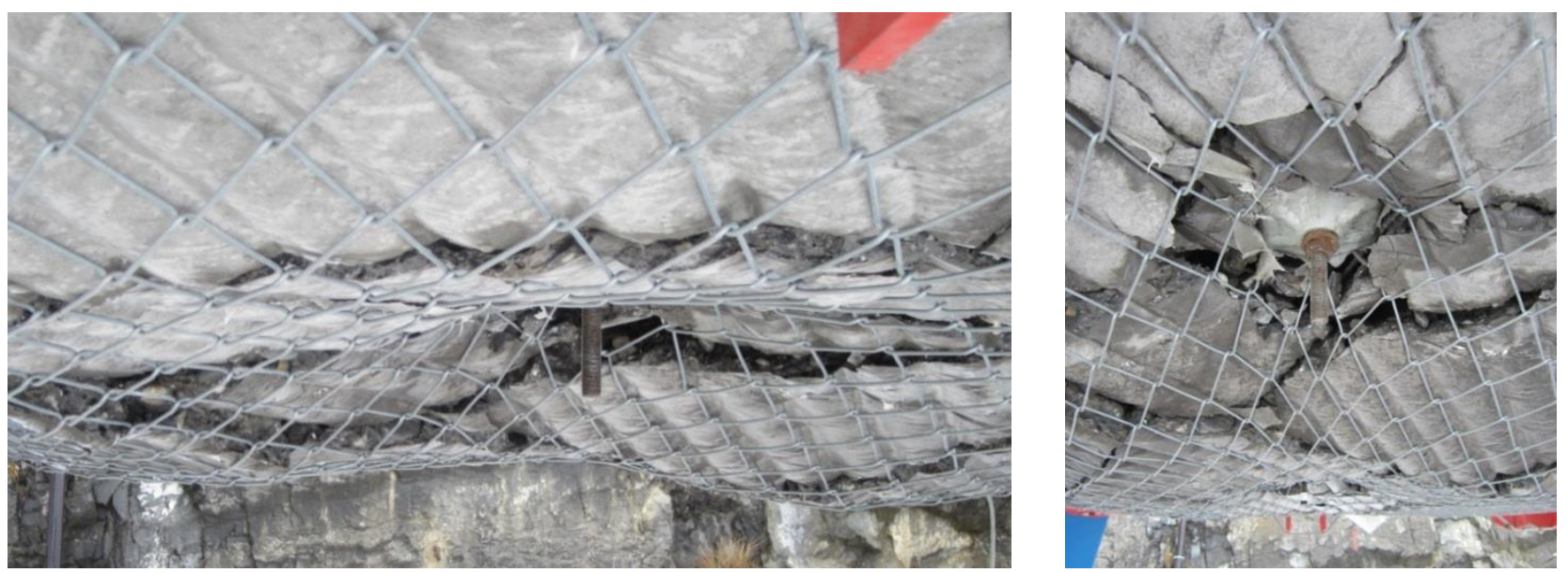

Figure 9 Deflection of the mesh for a softer assembly

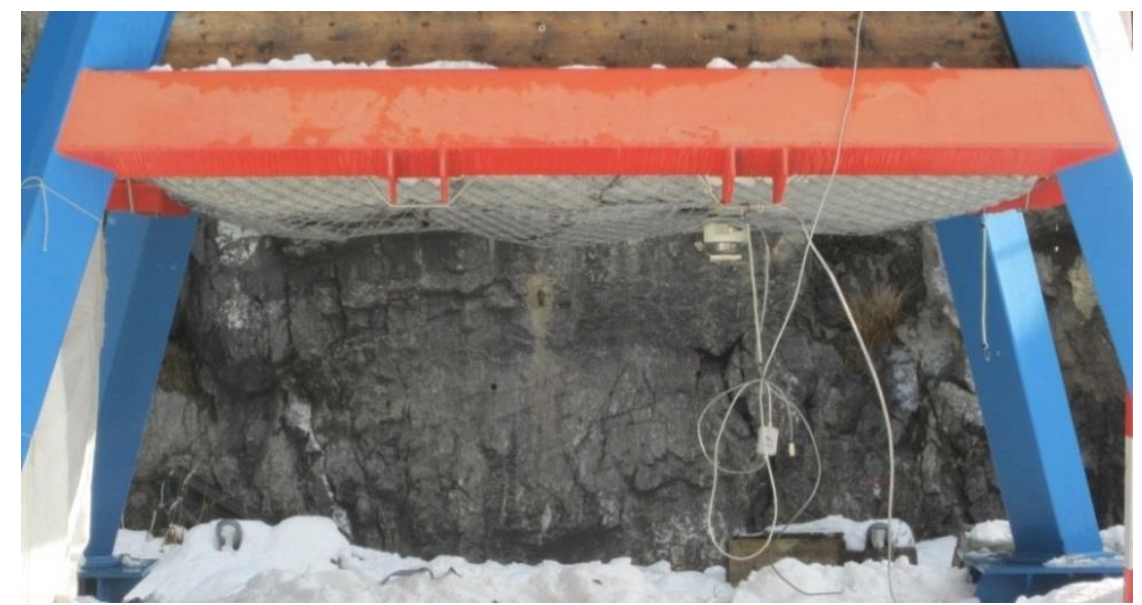

Figure 10 Loading of the mesh and transferring the loads to the plates 


\subsection{Test results}

Using a drop height of approximately $3.25 \mathrm{~m}$ and the test block's weight of $6,280 \mathrm{~kg}$, the energy input in the system can be calculated to $200 \mathrm{~kJ}$. Depending on the stiffness of the test set-up the energy absorbed by the mesh and the bolts will vary. The stiffer the test set-up, the more energy is absorbed by the bolts. This refers to rock types which do not break into small pieces during a seismic event. If they do so, then there is more load going into the mesh because these small blocks are ejected between the bolts into the mesh. Nevertheless, in both cases support elements have to be able to absorb their share of energy without rupture. Only the distribution of the energy absorption varies depending on the stiffness described above. This distribution is analysed in Section 3.5.

With regards to the energy absorbed by mesh and bolt it is necessary to have a look at the forces measured at the collar and the end of the bolt. The diagrams 11 and 12 show those forces for a stiffer and for a softer test set-up. The maximum force in the bolt for the stiffer test set-up has been about $240 \mathrm{kN}$. This value is located in the yielding area of the bolt. Regarding the mesh, the maximum measured force is about $65 \mathrm{kN}$. For this range of forces, no rupture of the mesh around the plates appears. Figure 11 shows the loads in the bolts during the stiff assembly event with time.

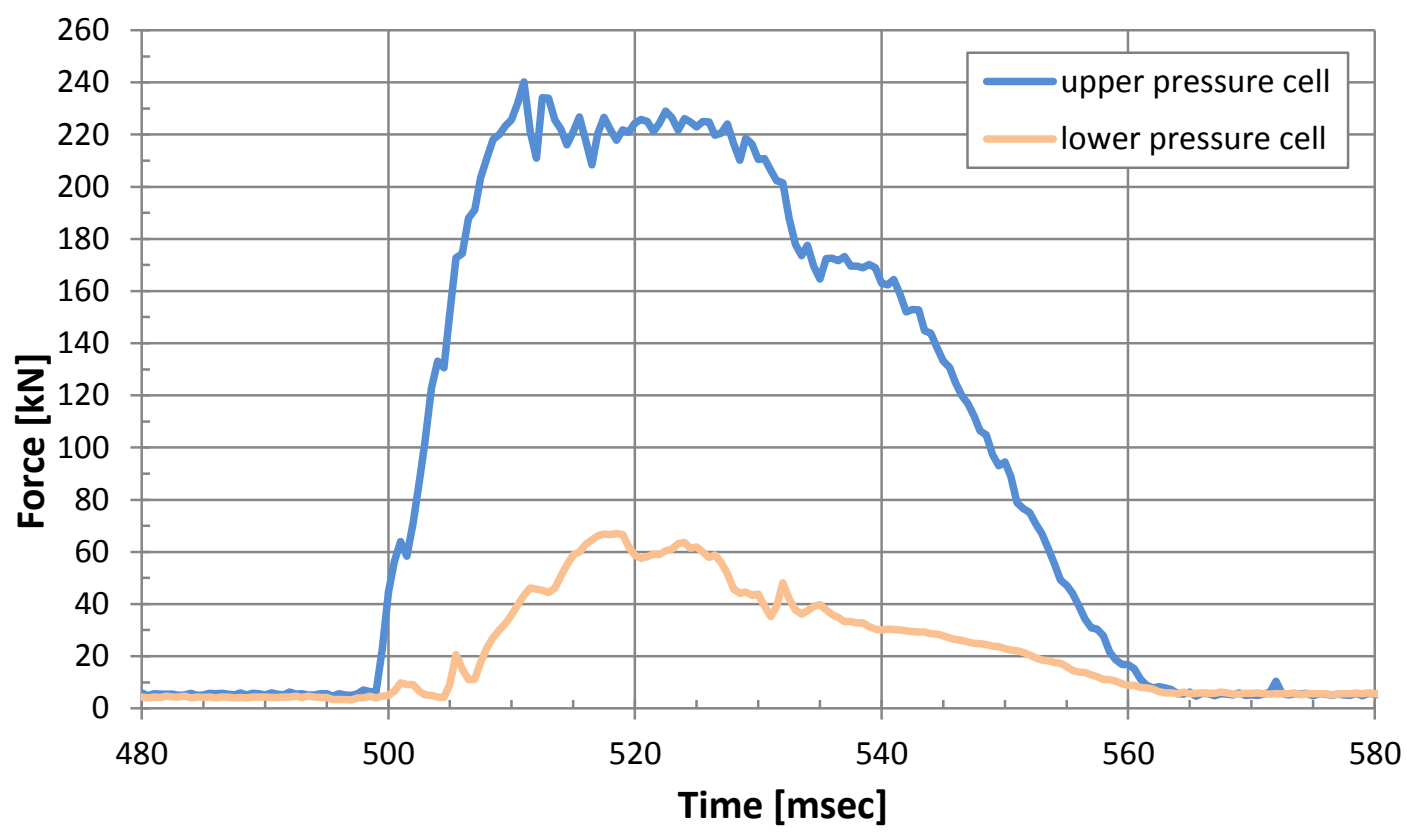

\section{Figure 11 Forces measured by the load cells for a stiff assembly}

For the softer test set-up, forces are generally lower and impact times longer caused by the smoother behaviour of the whole system. The maximum force measured by the upper load cell belongs to the dynamic bolt and its value is around $165 \mathrm{kN}$. This value is below the yielding load and the bolts were only lightly activated $(25 \mathrm{~mm})$. The maximum force from the mesh is about $50 \mathrm{kN}$. The forces for the soft assembly can be seen in Figure 12 . 


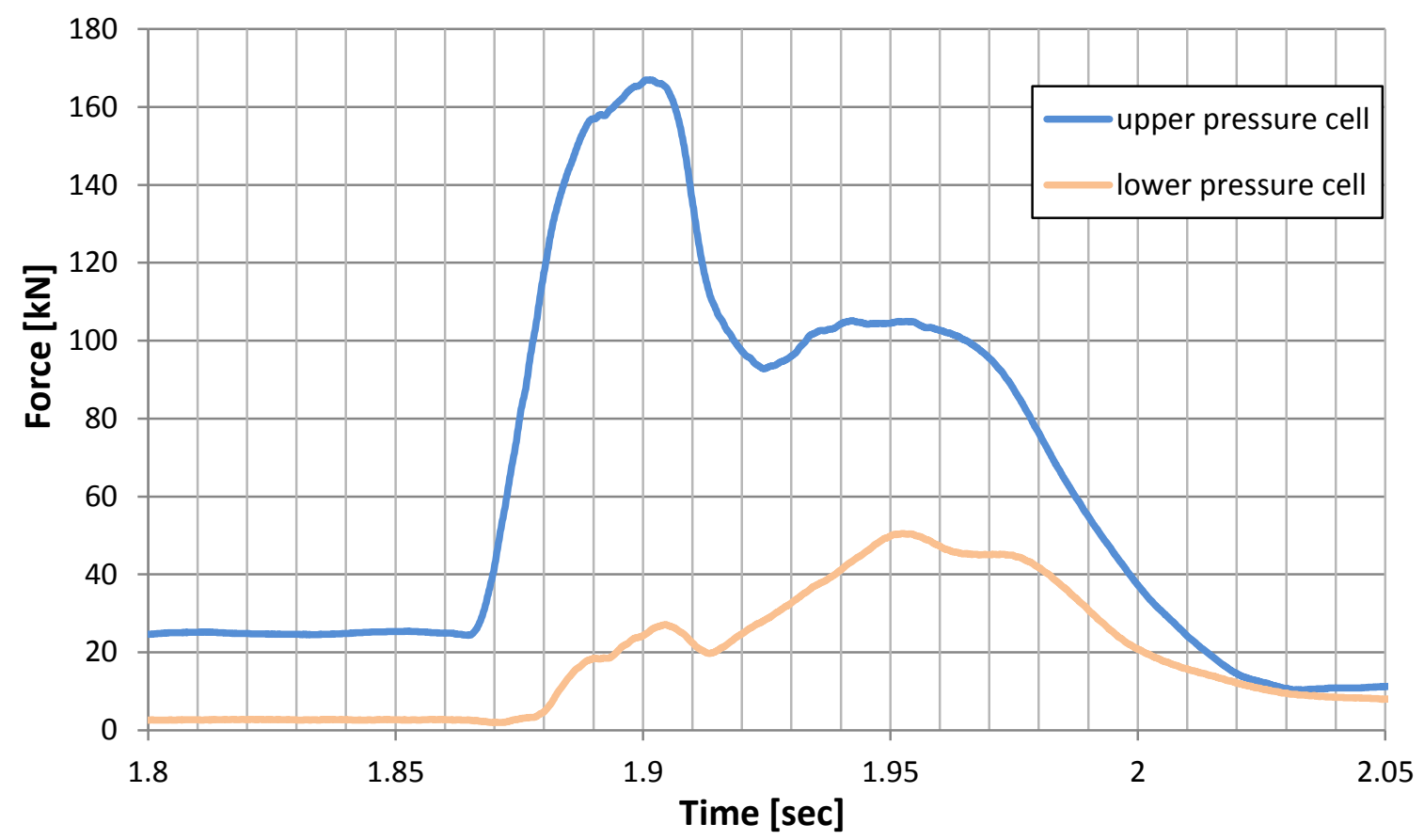

Figure 12 Forces measured by the load cells for a softer assembly

\subsection{Analysis}

As described above, the trials show that bolts and mesh work together, meaning that both support elements absorb a part of the energy without being overloaded. The amount of energy each part of the support will take depends on the type, stiffness and the behaviour of the rock mass.

The energy distribution in the stiff test set-up using the integration of the load-displacement has been back-calculated as follows. The energy absorbed by the bolts is about $32 \mathrm{~kJ}$ in total (about $8 \mathrm{~kJ}$ per bolt). The mesh absorbs about a third of this amount namely about $10 \mathrm{~kJ}$. In this setup a total of $42 \mathrm{~kJ}$ was absorbed by the support system where the bolts took $75 \%$ and the mesh $25 \%$.

Investigating the energy distribution of the softer set-up it can be seen that the bolts only dissipate a small amount of energy of about $10 \mathrm{~kJ}$ ( $2.5 \mathrm{~kJ}$ per bolt) while the mesh absorbs about $26 \mathrm{~kJ}$. In this case the mesh takes about $70 \%$ of the total energy of $36 \mathrm{~kJ}$ and the bolts only $30 \%$.

These two examples presented so far are rather extreme cases (although relatively small energies), with a very stiff and a very soft test set-up. The energy distribution for other cases will be in between these ratios. Based on the type of rock material and the magnitude of the seismic event the ratio will change. If the rock does not tend to break into small pieces during a rockburst and if the magnitude of the rockburst is big, then more energy will go onto the bolts. If this is not the case then more energy has to be absorbed by the mesh.

By knowing the energy dissipated by mesh and bolts, the energy absorbed by the test facility can be backcalculated. By subtracting the energy dissipated by bolts and mesh the left over amount of energy refers to the energy absorbed by the test set-up (in the above mentioned two cases about $160 \mathrm{~kJ}$ each).

\subsection{Energy distribution between mesh and bolts}

As shown in the sketch in Figure 13, the energy distribution on both support elements in regards to the stiffness of the test set-up can be described by a simple model. The distribution of the load between reinforcement and surface support could be described by using a ratio of the mass or energy distribution between mesh and bolts. The rock mass acting directly on the bolts will have a cone shape around the 
bolts. Between the bolts, arching effects are observed, meaning that the mass above the arch loads the bolt as well as the cone. The rock mass below the arch will then impact the surface support.

arching effect

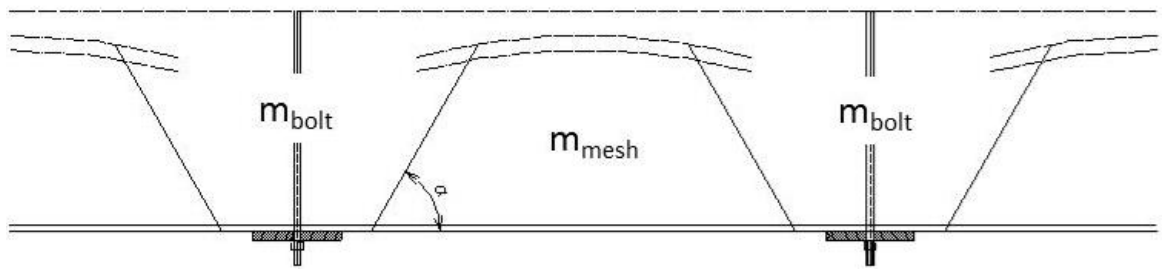

\section{Figure 13 Distribution of the activated rock mass on bolts and mesh}

For further consideration the ratio can be estimated by evaluating the properties and the behaviour of the rock mass. A stiffer rock mass which does not disintegrate much during a rockburst results in more mass and energy which have to be absorbed by the bolts. If the rock mass is breaking into quite small pieces then a bigger share of the mass and energy is going on the mesh. One has to keep in mind that rockburst events primarily occur in relatively high stressed brittle rock. Thus too heavily broken up rock mass is unlikely.

The results of the two presented tests suggest that in a rock mass where the rock is not breaking into small pieces an energy distribution of $25 \%$ mesh and $75 \%$ bolt can be assumed. If the rock breaks into smaller pieces then this can be the opposite: energy distribution of $70 \%$ mesh and $30 \%$ bolt. This ratio will also vary with the magnitude of the rockburst loading. The higher the loading the more mass has to be activated and the deeper the failure zone in the rock which consequently leads to an increase of the energy share for the bolts.

\section{$4 \quad$ Conclusions and outlook}

It can be concluded that the test site Walenstadt has been successfully commissioned and that it can be used to apply a dynamic rockburst loading to a larger panel of surface support and four bolts. The instrumentation with load cells, accelerometers and high speed video cameras does work as intended and allows an analysis and back calculation of the system behaviour and energy absorption. With the stiff setup it was possible to show that the test setup can impose enough energy to activate four strong dynamic bolts. With the soft setup, mainly the mesh was loaded and the anchors were lightly activated. This result will lead to the next step in subjecting the setup to higher energies and thus simulate stronger rockbursts which should lead to the loading of the mesh and the yielding of the dynamic bolts.

The test setup proved to be suitable to show the overall performance of such a ground support system. It was demonstrated that high-tensile mesh works together with the dynamic bolts. The method of connecting the two systems was shown to be suitable. However, this test cannot be compared to more repeatable lab testing since it includes breakage of the concrete slab and also the compaction of the rock mass. From that point of view it will be very interesting to test different rock mass setups and see the influence on energy distribution ratio between bolts and mesh. This will lead to a small database of such values which then may be compared to conditions in real applications.

An additional option would be to create a three-dimensional numerical model of the test facility. The database of previous experiments would serve to validate the numerical model. Then the simulation of other configurations of rock mass and support elements would be possible and reliable.

Based on this initial work it is hoped to be able to suggest a dimension factor for different ground conditions with a proven ratio of load which has to be absorbed by the surface support during a dynamic impact. This load then has to be transferred by the plate into the bolt without puncturing around the plate. Together with this information it will be possible to choose a dynamic bolt for the surface support which yields before the surface support is rupturing. These factors of the load distribution between mesh and bolts seem to be dependent on the rock type and the magnitude of the rockburst. 


\section{References}

Hadjigeorgiou, J. and Potvin, Y. (2011) A critical assessment of dynamic rock reinforcement and support testing facilities, Rock Mechanics and Rock Engineering, Springer Verlag, Vol. 44(5), pp. 565-578.

Heal, D., Hudyma, M. and Potvin, Y. (2004) Assessing the in-situ performance of ground support systems subjected to dynamic loading, in Proceedings Fifth International Symposium on Ground Support in Mining and Underground Construction, E. Villaescusa and Y. Potvin (eds), 28-30 September 2004, Perth, Australia, Balkema, Rotterdam, pp. 319-326.

Heal, D. (2010) Observations and analyses of incidences of rock burst damage in underground mines, PhD thesis, University of Western Australia, Perth.

Kaiser, P.K., McCreath, D.R. and Tannant, D.D. (1995) Canadian Rockburst Research Program. Vol. 2, Camiro Mining Division, Sudbury, Ontario, Canada.

Player, J.R., Villaescusa, E. and Thompson, A.G. (2004) Dynamic testing of rock reinforcement using the momentum transfer concept, in Proceedings Fifth International Symposium on Ground Support in Mining and Underground Construction, E. Villaescusa and Y. Potvin (eds), 28-30 September 2004, Perth, Australia, Balkema, Rotterdam, pp. 327-339.

Potvin, Y. (2012) An interpretation of ground support capacity submitted to dynamic loading, Newsletter Australian Centre for Geomechanics, Australian Centre for Geomechanics, Perth, Australia, Vol. 39, December 2012, pp. 1-5.

Thompson, A.G., Villaescusa, E. and Windsor, C.R. (1/3/2012) Ground support terminology and classification: an update, Springer International Publishing AG, viewed 12 April 2013, http://link.springer.com/article/10.1007/s10706-012-9495-4. 